\title{
NUMERICAL INVESTIGATION OF NATURAL CONVECTION WITH HEATED TUBES IN TUNNEL GREENHOUSE
}

\author{
Y. Slatni ${ }^{1}$, M. Djezzar ${ }^{1}$, T. Messai ${ }^{2}$
}

\begin{abstract}
In this research, a numerical study was carried out on heat transfer by natural convection, in a closed tunnel greenhouse, in the range of the Rayleigh number $\left(10^{3} \leq \mathrm{Ra} \leq 10^{6}\right)$. Were considered in the study, the number of heating tubes used $(1 \leq \mathrm{N} t \leq 7)$, which were equidistant inside the greenhouse volume, when the bottom at an average temperature and cold Roof. The governing equations written in a bicylindrical coordinates were discretized using the finite volume method and vorticity-stream function formulation; the resulting algebraic equations were solved using successive over relaxation method (S.O.R). First, the effect of the Rayleigh number on heat transfer was examined for a fixed number of tubes as reference $(\mathrm{N} t=3)$ and the number of tubes was varied to investigate the influence on heat transfer in the greenhouse. Finally, the results obtained were summarized in the form of isotherms and streamlines, and for the average Nusselt number profile; in addition to the horizontal and vertical velocities and temperatures. However, in the reference case, for low Rayleigh numbers, the heat transfer is dominated by pure conduction. With the increase of the Rayleigh number and the number of tubes $\mathrm{N} t$, the natural convection becomes more dominant and the heat transfer increases, and in general the heat transfer increase with the increasing number of tubes.
\end{abstract}

Keywords: Natural Convection, Bicylindrical Cordinates, Heating Tubes, Tunnel Greenhouse, The Vorticity-Streamfunction Formulation, S.O.R Method

\section{INTRODUCTION}

The tunnel greenhouse is a production tool, allowing to create artificially a microclimate intended to favor the growth and the development of cultures. The main climatic factors of the internal environment in a greenhouse, which are different with regard to the outside, are: the temperature, the light and the humidity. In this system, the natural convection is a particularly important mechanism, for heat exchange between the internal air and all other solid surfaces (Bottom, walls, roof...). The effect of the geometric form has been studied in many numerical and experimental works using various geometric forms.

A various numerical and experimental studies dealing with the problem of heating and natural convection in greenhouses were carried out. One of these studies was that of U-Cheul Shin et al. [1] who conducted a theoretical study on the natural convection in air-filled inclined enclosure. Has primarily focused on the influence of the various parameters of the system, in particular the inclination with respect to the horizontal plane, the shape factor, the number of Grashof, the average and local values of the Nusselt numbers, as well as temperature and streamlines distributions of. He has find that the minimum heat transfer is obtained for angles of inclination between 30 and $45^{\circ}$. For large values of the Grashof number, the transfer is accelerated because of secondary flows. Draoui et al. [2-3] conducted a numerical simulation in laminar unsteady natural Convection in tunnel and mono-chapel greenhouse heated by the bottom. The results are given in the form of distributions of isotherms, streamlines and local Nusselt number Together with the variation of the temperature in the center of the greenhouse as a function of time. The results obtained show that presence of two counter-rotating vortices, of large sizes located on either side of the vertical median plane, as well as the pressure of the air inside the greenhouse is important near the ground, this allows a good air circulationand supports the idea of putting the openers up. Bondareva et al. [4] inspected This paper was recommended for publication in revised form by Regional Editor Tolga Taner ${ }^{1}$ Energetic Physics Laboratory, Exact Sciences Faculty, Frères Mentouri Constantine 1 University, Algeria ${ }^{2}$ Department of Mechanical Engineering, Akli Mohand Oulhadj University, Bouira, Algeria *E-mail address: slatniyacine@gmail.com,mdjezzar@yahoo.fr, messi.tarek@gmail.com Orcid id: 0000-0003-1559-6428, 0000-0001-6532-9964, 0000-0002-2916-2498

Manuscript Received 29 March 2019, Accepted 06 December 2019 
numerically a natural convection in different enclosures. The results are given in the form of distributions of isotherms, streamlines and local Nusselt number, also Profiles of vertical velocity and temperature. Where found the better solution for the heating and cooling of electronic devices, as well as thermal energy storage systems. Altaç et al. [5] have investigated numerically a transient analysis of flow and heat transfer in 2D and 3D for different domed and curved structures. Streamlines and isotherms are displayed, also the calculation of the average Nusselt number near to the hot wall, where the results are obtained for different geometries. The average Nusselt number in the 2D enclosure does not change significantly with the change in the size of the cavity. On the other hand, average Nusselt higher in 3D calculations, but still they are smaller compared to flat top enclosures. Mezrhab et al. [6] reported on the computation of surface radiation and natural convection in a heated horticultural greenhouse. Results were reported in terms of isotherms, streamlines and average Nusselt number for Rayleigh number in the range $10^{3}-10^{6}$. The isotherm lines show that the radiative effects are observed near the solid surfaces, and become increasingly important when the Rayleigh number and the horizontal velocity profile increase. Çerçi et al. [7] mentioned an experimental study for drying apple slices in direct sunlight and calculated the heat transfer coefficient. Shows that a low value of the average coefficient of heat transfer by convection was found in the greenhouse during drying by natural convection, on the other hand the highest value was shown in drying in the open sun. As welle as notes that the heat transfer coefficient influences by many parameters such as product size, porosity, humidity, thermo-physical properties and climatic conditions. Oztop et al. [8] studied The récents studies of natural convection calculation methods for complicated shapes. The results obtained that the geometry parameter is an important control parameter on heat and fluid flow in natural convection; also they have shown that the presence of obstacles will degrade the heat transfer process and this with higher values of the external Rayleigh number. The local and average Nusselt number decreases with increasing values of the internal Rayleigh number and the Hartmann number. Kabdi et al. [9] made a numerical study of two-dimensional laminar steady natural convection in cylindrical lunulas. Their results show that with a suitable choice of parameters, it is possible to control the natural convection by favoring, for instance, a definite way of heat transfer (conductive or convective). When the plane of the axes of the two cylinders is inclined, a secondary flow is seen in the upper part of the lunula. On the other hand, if the axes plane is vertical, no secondary flow is observed. If the lunula is filled with a porous medium saturated with fluid, the transfer model is based on Darcy's law. Chin-Lung et al. [10] realized an experimental and numerical study of mixed convection and flow pattern in a lid-driven arc-shape cavity. They studied the effect of Grashof and Reynolds numbers in the form of isotherms and streamlines along with the local and average Nusselt number. Results show that only when the Grashof number is $>10^{5}$, the increase in natural convection heat transfer becomes appreciable. The vortex strength and pattern are found to be dependent on the inclination angle. At $\theta=0$ or $\Pi$, a flow pattern of two symmetric cells is found, whereas at other inclination angles the flow field features a one-cell pattern and a thermal boundary is formed along the flat wall. Bartzanas et al. [11] reported the results of the Influence of the heating method on greenhouse microclimate and energy consumption. Simulation results indicate that the use of a mixed system is favourable in greenhouse tunnel conditions since the use of the air heater, although increasing slightly the energy consumption, improves the control of both air temperature and humidity, particularly by keeping the inside air dew point temperature lower than the cover temperature and preventing the occurrence of condensation on the plastic films. Tadj [12] studied a numerical calculation of convection in horticultural greenhouse heating. The results are represented in the form of streamlines, isotherms and velocities profiles, tried to find the optimal solution for the thermal design of the greenhouse, as well as the best position of the air conditioning system. Dihmani et al. [13] investigated numerically the thermal transfers in an agricultural greenhouse heated by isothermal solid blocks. The results are presented in terms of isotherms, streamlines and Nusselt numbers for Rayleigh numbers between $10^{3}-10^{6}$. They concluded that the influence of thermal radiation is all the more important as the number of Rayleigh is high. The results show that solid blocks promote heat transfer in the greenhouse. Y1ldiz [14] studied the cooling of a plate ( $1 \mathrm{~m}$ in height and $1 \mathrm{~m}$ in width), which is oriented vertical, and the results show that the surface temperatures of the plate at the inclination angle of $45^{\circ}$ are relatively higher than those of the plate at the vertical state and those of the $20^{\circ}$ inclined plate. Aich et al. [15] studied numerically three-dimensional computational fluid dynamics analysis of buoyancy-driven natural ventilation and entropy generation in a prismatic greenhouse. Results are reported in terms of particles trajectories, iso-surfaces of temperature, mean Nusselt number, and entropy generation. It was found that 
the flow structure is sensitive to the value of Rayleigh number and that heat transfer increases with increasing this parameter. Furthermore, using asymmetric opening positions improves the natural venti-lation and facilitates buoyancy induced upward cross air-flow (low-level supply and upper-level extraction) inside the greenhouse. In the study of Yejjer et al. [16] concerning three-dimensional natural convection and entropy generation in an inclined solar collector equipped with partitions, results are reported in terms of isosurfaces of temperature, isotherms, particles trajectories, velocity vector projection, average Nusselt number along the absorber plate and entropies generation contours. Aich et al. [17] made a numerical analysis of natural convection in parismatic enclosure that looks like a monochapel greenhouse; he found that heat transfer increases with increasing Rayleigh number and decreases with increasing aspect ratio.

Based on the literature search mentioned above, it appears that the numerical study of natural convection in the greenhouse heated by the tubes has not been approached in detail, tunnel or chapel geometries. In other words, most studies on natural convection do not use heating of greenhouses with heating tubes, as a means of effectively harnessing geothermal energy for heating at night or on cloudy days in cold weather. For these reasons, and to be more effective for the study, we primarily looked at the effects of the Rayleigh number and the Nt number of tubes on fluid flow and heat transfer properties in a greenhouse. So, streamlines, isotherms together with average and local Nusselt numbers are illustrated through key parameters such as the Rayleigh number and the number of tubes.

\section{MATHEMATICAL MODEL}

We consider the natural convection of an incompressible fluid in a tunnel greenhouse with height $K$ and width $L$ (see Fig. 1.). The considered problem is a two-dimensional heat transfer inside a greenhouse, with a hot tubes at constant temperature $T_{H}$, a bottom at an average temperature $T_{M}$ and a cold roof with $T_{C}$, when $T_{H}>T_{M}>T_{C}$. The flow is assumed: incompressible, laminar, steady and the Boussinesq approximation is adopted in buoyancy force such:

$$
\rho=\rho_{0}\left(1-\beta\left(T-T_{C}\right)\right)
$$

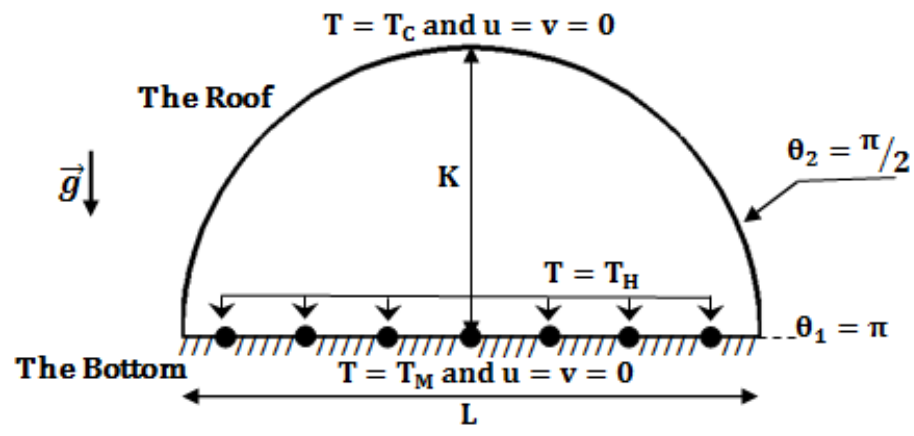

Figure 1. Schematic diagram of the physical system

\section{General equations}

In our study, it is assumed that the medium is continuous and isotropic and that there is no source or sink of matter so that the equations we have are the following:

$$
\begin{aligned}
& \frac{\partial \rho}{\partial t}+\nabla \rho \vec{V}=0 \\
& \frac{d \vec{V}}{d t}=\rho \vec{f}-\vec{\nabla} P+\vec{\nabla} \tau
\end{aligned}
$$


Journal of Thermal Engineering, Research Article, Vol. 7, No. 4, pp. 731-745, May, 2021

$$
\frac{d}{d t}\left(\rho c_{p} T\right)=\vec{\nabla}(\lambda \vec{\nabla} T)+T \beta \frac{d P}{d t}+\mu \phi+q
$$

The governing equations for laminar steady convection, after invoking the Boussinesq approximation and neglecting the viscous dissipation, can be expressed in the vorticity-stream function formulation as:

$$
\begin{aligned}
& \frac{\partial^{2} \psi}{\partial x^{2}}+\frac{\partial^{2} \psi}{\partial y^{2}}=-\omega \\
& u \frac{\partial T}{\partial x}+v \frac{\partial T}{\partial y}=\alpha\left(\frac{\partial^{2} T}{\partial x^{2}}+\frac{\partial^{2} T}{\partial y^{2}}\right) \\
& u \frac{\partial \omega}{\partial x}+v \frac{\partial \omega}{\partial y}=g \beta \frac{\partial T}{\partial x}+v\left(\frac{\partial^{2} \omega}{\partial x^{2}}+\frac{\partial^{2} \omega}{\partial y^{2}}\right)
\end{aligned}
$$

With:

$$
\omega=\frac{\partial v}{\partial x}-\frac{\partial u}{\partial y}, u=\frac{\partial \psi}{\partial y}, \quad v=-\frac{\partial \psi}{\partial x}
$$

The transformation between the Cartesian coordinates $(x, y)$, and the Bicylindrical coordinates $(\eta, \theta)$ is obtained by the following relations:

$$
\left\{\begin{array}{l}
x=\frac{a \cdot \sinh (\eta)}{(\cosh (\eta)-\cos (\theta))} \\
y=\frac{a \cdot \sin (\theta)}{(\cosh (\eta)-\cos (\theta))}
\end{array}\right.
$$

After using the coordinate transformation the governing equations $(5,6$, and 7$)$ becomes:

$$
\begin{gathered}
\frac{\partial^{2} \psi}{\partial x^{2}}+\frac{\partial^{2} \psi}{\partial y^{2}}=-h^{2} \omega \\
V_{\eta} \frac{\partial T}{\partial \eta}+V_{\theta} \frac{\partial T}{\partial \theta}=\frac{1}{h}\left(\frac{\partial^{2} T}{\partial x^{2}}+\frac{\partial^{2} T}{\partial y^{2}}\right) \\
V_{\eta} \frac{\partial \omega}{\partial \eta}+V_{\theta} \frac{\partial \omega}{\partial \theta}=\frac{v}{h}\left(\frac{\partial^{2} \omega}{\partial \eta^{2}}+\frac{\partial^{2} \omega}{\partial \theta^{2}}\right)+g \beta\left(F(\eta, \theta) \frac{\partial T}{\partial \eta}-G(\eta, \theta) \frac{\partial T}{\partial \theta}\right)
\end{gathered}
$$

With:

$$
\left\{\begin{array}{c}
V_{\eta}=\frac{1}{h} \frac{\partial \psi}{\partial \theta} \\
V_{\theta}=-\frac{1}{h} \frac{\partial \psi}{\partial \eta}
\end{array}, \omega=-\frac{1}{h^{2}}\left(\frac{\partial^{2} \psi}{\partial \eta^{2}}+\frac{\partial^{2} \psi}{\partial \theta^{2}}\right)\right.
$$


And

$$
\begin{gathered}
h=\frac{a}{(\cosh (\eta)-\cos (\theta))} \\
\left\{\begin{array}{l}
F(\eta, \theta)=\frac{1-\cosh (\eta) \cos (\theta)}{(\cosh (\eta)-\cos (\theta))} \\
G(\eta, \theta)=\frac{1-\sinh (\eta) \sin (\theta)}{(\cosh (\eta)-\cos (\theta))}
\end{array}\right.
\end{gathered}
$$

Introducing the following dimensionless variables:

$$
D_{h}=a ; H=\frac{h}{D_{h}} ; V_{\eta}^{*}=V_{\eta} \frac{D_{h}}{\alpha} ; V_{\theta}^{*}=V_{\theta} \frac{D_{h}}{\alpha} ; \psi^{*}=\frac{\psi}{\alpha} ; \omega^{*}=\omega \frac{D_{h}^{2}}{\alpha} ; \text { and } T^{*}=\frac{T-T_{f}}{T_{c}-T_{f}}
$$

Then the equations (10), (11) and (12) becomes:

$$
\begin{gathered}
\frac{\partial^{2} \psi^{*}}{\partial \eta^{2}}+\frac{\partial^{2} \psi^{*}}{\partial \theta^{2}}=-H^{2} \omega^{*} \\
V_{\eta}^{*} \frac{\partial T^{*}}{\partial \eta}+V_{\theta}^{*} \frac{\partial T^{*}}{\partial \theta}=\frac{1}{H}\left(\frac{\partial^{2} T^{*}}{\partial \eta^{2}}+\frac{\partial^{2} T^{*}}{\partial \theta^{2}}\right) \\
V_{\eta}^{*} \frac{\partial \omega^{*}}{\partial \eta}+V_{\theta}^{*} \frac{\partial \omega^{*}}{\partial \theta}=\frac{\operatorname{Pr}}{H}\left(\frac{\partial^{2} \omega^{*}}{\partial \eta^{2}}+\frac{\partial^{2} \omega^{*}}{\partial \theta^{2}}\right)+\operatorname{Pr} \cdot \operatorname{Ra}\left(F(\eta, \theta) \frac{\partial T^{*}}{\partial \eta}-G(\eta, \theta) \frac{\partial T^{*}}{\partial \theta}\right)
\end{gathered}
$$

\section{The dimensionless boundary conditions}

At the heating tubes:

Outside heating tubes:

$$
V_{\eta}^{*}=V_{\theta}^{*}=\frac{\partial \psi^{*}}{\partial \eta}=\frac{\partial \psi^{*}}{\partial \theta}=0 ; \omega^{*}=-\frac{1}{H^{2}}\left(\frac{\partial^{2} \omega^{*}}{\partial \eta^{2}}+\frac{\partial^{2} \omega^{*}}{\partial \theta^{2}}\right)=0 ; T=T_{H}
$$

$$
V_{\eta}^{*}=V_{\theta}^{*}=\frac{\partial \psi^{*}}{\partial \eta}=\frac{\partial \psi^{*}}{\partial \theta}=0 ; \omega^{*}=-\frac{1}{H^{2}}\left(\frac{\partial^{2} \omega^{*}}{\partial \eta^{2}}+\frac{\partial^{2} \omega^{*}}{\partial \theta^{2}}\right)=0 ; T=T_{M}
$$

At the roof:

$$
V_{\eta}^{*}=V_{\theta}^{*}=\frac{\partial \psi^{*}}{\partial \eta}=\frac{\partial \psi^{*}}{\partial \theta}=0 ; \omega^{*}=-\frac{1}{H^{2}}\left(\frac{\partial^{2} \omega^{*}}{\partial \eta^{2}}+\frac{\partial^{2} \omega^{*}}{\partial \theta^{2}}\right)=0 ; T=T_{C}
$$

\section{Average Nusselt number Calculation}

The average Nusselt numbers is expressed and calculated by the sum of heat fluxes on the bottom wall from the following relation:

$$
N u_{a v}=\frac{1}{\left(\eta_{N I}-\eta_{1}\right)} \int_{\eta_{1}}^{\eta_{N I}} N u \partial \eta=\frac{1}{\left(\eta_{N I}-\eta_{1}\right)} \sum_{i=1}^{i=N I} N u_{i}
$$


Where $N u$ is the local Nusselt number, defined as:

$$
N u_{l}=-\frac{1}{H}\left(\frac{\partial T^{*}}{\partial \theta}\right)_{\theta=\theta_{1}}
$$

\section{NUMERICAL METHOD AND VALIDATION}

The greenhouse system is illustrated in figure (2), with the physical domain representing the real case and the computation domain representing the calculation domain. The governing equations (16) to (18), with the corresponding boundary conditions given in (eqs.19, 20 and 21) are discretized using the finite volume method [18] with non-staggered grid. The diffusion term in the vorticity and energy equations is approximated by a central differencing scheme (CDS), and power-law differencing scheme (PLDS) was adopted for the convective terms [19].

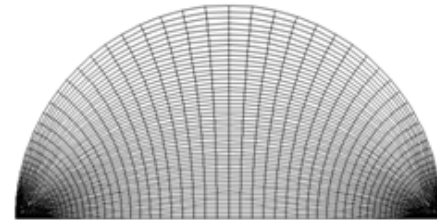

Physical domain

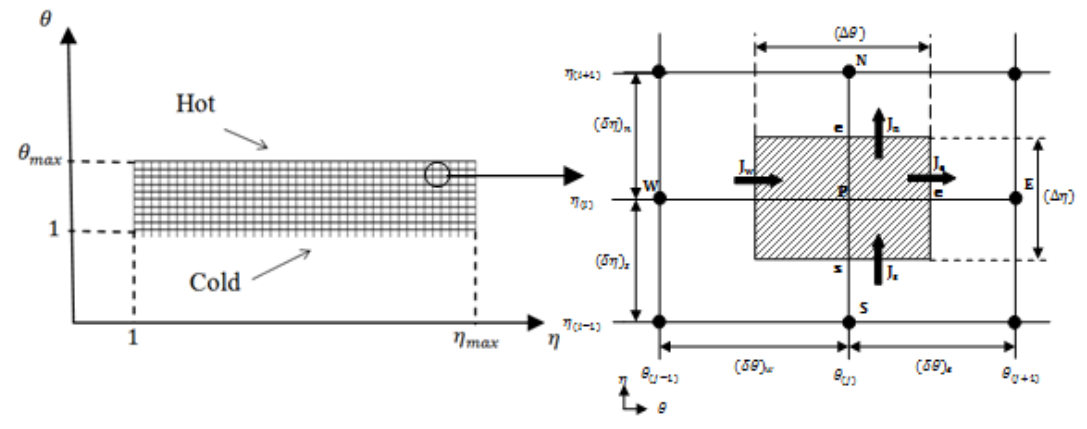

Computation domain
Typical control volume

Figure 2. Physical and computation domain

The algebraic equations for the vorticity-stream function and energy equations are written into the following form:

$$
a_{P} \phi_{P}=a_{E} \phi_{E}+a_{W} \phi_{W}+a_{N} \phi_{N}+a_{S} \phi_{S}+b
$$

Where the subscripts P, W, E, N and S denote cell location, west, east, north and south face of the volume control, respectively. Also, the symbol $\phi$ denotes any scalar transport quantity namely: $\psi, \omega$ or $\mathrm{T}$ when $b$ corresponding the source term. The resulting algebraic equations are solved using successive over relaxation method (SOR) [20]. To accelerate the convergence, an under-relaxation factor is used for all variables: $\psi$, $\omega$ or T The convergence criterion is defined by the following expression:

$$
\varepsilon=\left|\frac{\max \phi^{n+1}-\max \phi^{n}}{\max \phi^{n}}\right| \leq 10^{-8}
$$

Where $\varepsilon$ is the tolerance of convergence and $n$ the number of iterations.

\section{Grid independence study}

In the present paper, a uniform grid was used. Numerical experiments were performed in order to check the grid independence of the solutions. The grid sizes are tested from $29 \times 11$ to $119 \times 55$ for Ra $=10^{5}$ and $10^{6}$; the Table 1 shows the convergence of the maximum stream function value. It is observed from the grid independent study that a $119 \times 55$ grid is sufficient to obtain a numerical solution. 
Journal of Thermal Engineering, Research Article, Vol. 7, No. 4, pp. 731-745, May, 2021

Table 1. Grid independency test for the maximum streamlines values at $\mathrm{Ra}=10^{5}, 10^{6}$

\begin{tabular}{|c|c|c|c|c|c|c|c|c|c|c|c|}
\hline $\mathbf{R a}$ & Grid & $29 \times 11$ & $39 \times 15$ & $49 \times 21$ & $59 \times 25$ & $69 \times 31$ & $79 \times 35$ & $89 \times 41$ & $99 \times 45$ & $109 \times 51$ & $119 \times 55$ \\
\hline \multirow{2}{*}{$10^{5}$} & $\psi_{\max }$ & 12.14 & 12.59 & 13.13 & 13.30 & 13.57 & 13.73 & 13.84 & 13.91 & 13.94 & 13.96 \\
\hline & $E_{r} \%$ & -- & 3.70 & 4.29 & 1.29 & 2.03 & 1.17 & 0.80 & 0.51 & 0.21 & 0.14 \\
\hline \multirow{2}{*}{$10^{6}$} & $\psi_{\max }$ & 37.26 & 39.69 & 40.62 & 41.93 & 42.52 & 43.35 & 43.64 & 44.20 & 44.47 & 44.29 \\
\hline & $E_{r} \%$ & -- & 6.52 & 2.34 & 3.22 & 1.41 & 1.95 & 0.67 & 1.28 & 0.61 & 0.56 \\
\hline
\end{tabular}

Code validation

The numerical program developed in the present study was validated by comparing the results obtained between our results and those of Kabdi et al [9] while taking into consideration the boundary conditions with heat flux at the bottom wall and cold wall temperature at the roof, with various factor form $\left(f=r_{1} / r_{2}\right)$. A comparison of the average Nusselt number for the Grashof number range $\left(5 \leq G r \leq 2.10^{6}\right)$, predicted by Kabdi et al [9], with that of this study, as shown in Table 2 where an excellent agreement is found.

Table 2. Comparison of average Nusselt number of Reference [9] with our results

\begin{tabular}{|c|c|c|c|c|}
\hline \multirow{2}{*}{$\boldsymbol{f} \boldsymbol{f}$} & \multicolumn{2}{|c|}{$\boldsymbol{f}=\mathbf{0 . 5}$} & \multicolumn{2}{c|}{$\boldsymbol{f = 0 . 6}$} \\
\cline { 2 - 5 } & Our code & Ref.[9] & Our code & Ref.[9] \\
\hline $\mathbf{5}$ & 5.2910 & 5.286 & 5.2910 & 5.286 \\
\hline $\mathbf{1 0}$ & 5.2910 & 5.286 & 5.2910 & 5.286 \\
\hline $\mathbf{7 0}$ & 5.2910 & 5.286 & 5.2910 & 5.286 \\
\hline $\mathbf{1 0}^{\mathbf{2}}$ & 5.2910 & 5.286 & 5.2910 & 5.286 \\
\hline $\mathbf{7 . 1 0}^{\mathbf{2}}$ & 5.2912 & 5.287 & 5.2912 & 5.287 \\
\hline $\mathbf{1 0}^{\mathbf{3}}$ & 5.2914 & 5.287 & 5.2914 & 5.287 \\
\hline $\mathbf{1 . 4 . 1 0}^{\mathbf{4}}$ & 5.3555 & 5.357 & 5.3555 & 5.357 \\
\hline $\mathbf{2 . 1 0}^{\mathbf{4}}$ & 5.3575 & 5.357 & 5.3575 & 5.357 \\
\hline $\mathbf{1 . 4 . 1 0}^{\mathbf{6}}$ & 9.9629 & 9.969 & 9.9629 & 9.969 \\
\hline $\mathbf{2 . 1 0}$ & 9.4368 & 9.438 & 9.4368 & 9.438 \\
\hline
\end{tabular}

\section{RESULTS AND DISCUSSION}

The effect of natural convection with the buoyancy force in the greenhouse has a great importance in the research domain. For this section, a numerical investigation is made on the natural convection in the greenhouse, and the fluid is chosen as air with a Prandtl number $P r=0.702$. All computations were performed in the range of Rayleigh number $\left(10^{3} \leq R a \leq 10^{6}\right)$ and the spatial mesh $(119 \times 55)$ was used.

The objective of this study is to examine the influence of the Rayleigh number and the number of tubes $\mathrm{N} t$ on the flow and the heat transfer inside the greenhouse. The Results are presented in the form of streamlines and isothermal lines, as well as in terms of variations in the average Nusselt number and the temperature distribution, in four chosen positions inside the greenhouse.

\section{Dynamic field}

For the dynamic field, the streamlines are provided in Figure 3 (right), where the flow is controlled by two main symmetrical cells rotating in opposite senses, for different values of Rayleigh number. For $\mathrm{Ra}=10^{3}$ figure, two large sized counter-rotating vortex, located in the heart of the greenhouse, can be observed. On the left side the flow rotates clockwise but on the right side, it is anticlockwise because of the symmetrical greenhouse structure, where the fluid particles move upwards, under the action of the Archimedes forces. It can be said that the heat transfer occurs by pseudo-conduction, where the velocity fields are different from zero. The streamlines values given in this case are very small. As for $\mathrm{Ra}=10^{4}$, it is shown that the streamlines are modified sensibly, where the centers of the two vortex move slightly upwards to the middle of the greenhouse, and increases in streamlines values appear on the same figure compared to the previous case. This leads to a transformation of the conductive heat transfer to a convective 
heat transfer, but which remains relatively weak. When $\mathrm{Ra}=10^{5}$ and $10^{6}$, the centers of the two vortices move upwards, the maximum value of the current function increases significantly, and the shape of the geometry adopted by the isotherms, denotes an intensification of the flow, so we are in the presence of a natural convection casting in the greenhouse.

$\mathbf{R a}$

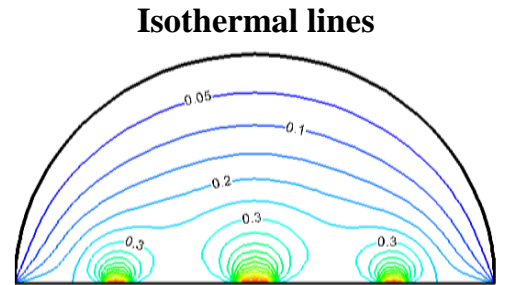

$10^{3}$

\section{3}

$0^{4}$

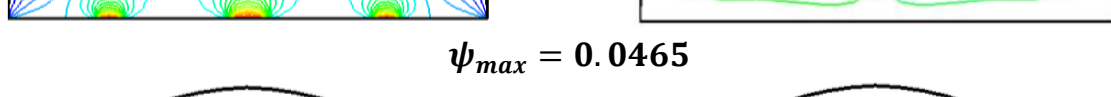

$10^{4}$

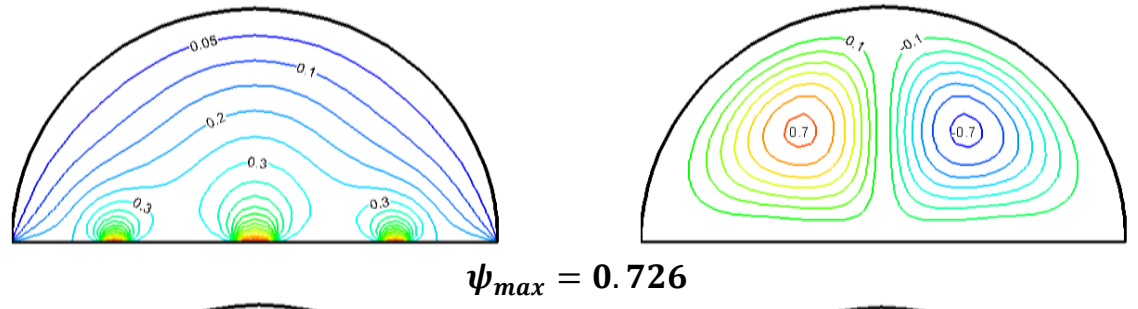

$10^{5}$
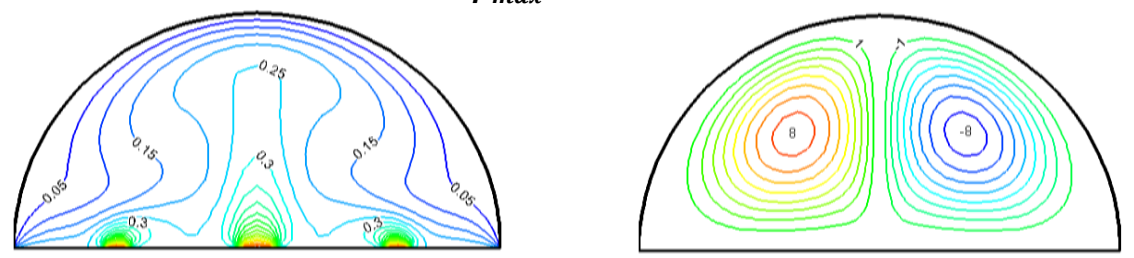

$\psi_{\max }=8.506$

$10^{6}$
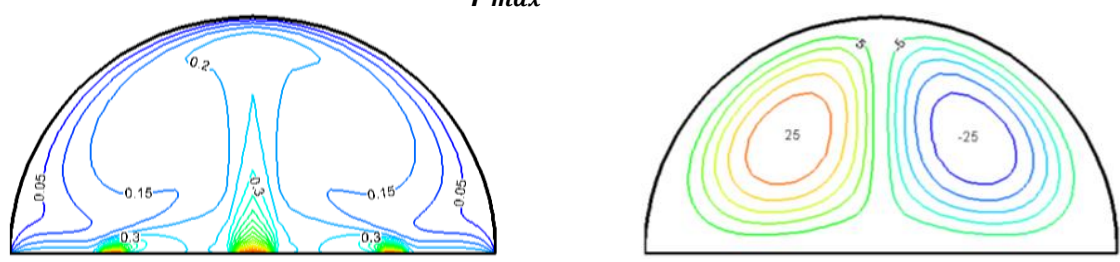

$$
\psi_{\text {max }}=30.01
$$

Figure 3. Isothermal lines and streamlines for three tubes in different values of Rayleigh number

\section{Thermal field}

As shown in Figure 3 (left), for $\mathrm{Ra}=10^{3}$, the isothermal lines are almost parallel curves and take the roof shape; in this case, the temperature distribution is simply decreasing from the bottom to the roof. So, in this case, the control mode is conduction. For $\mathrm{Ra}=10^{4}$, the isothermal lines are appreciably modified, this translates into a convective heat transfer mode. However, for $\mathrm{Ra}=10^{5}$ and $10^{6}$, represented respectively, the isotherm changes and take a mushroom form following the rotation direction of the streamlines whereas for $\mathrm{Ra}=10^{6}$, the form of mushroom becomes more intense and occupies a large part of the greenhouse, which translates the dominance of convection.

\section{Temperature and velocity distribution}

In this part, distribution of temperature and velocity within the greenhouse are presented, for that purpose, three vertical axes $\mathrm{A}, \mathrm{B}$ and $\mathrm{C}$ were traced together with three horizontal axes $\mathrm{D}, \mathrm{E}$ and $\mathrm{F}$, both in the points $1 / 2,3 / 8$ and $1 / 4$. Figures 4 and 5 show temperature and velocity distribution along the three axes in the greenhouse for $\mathrm{Ra}=10^{3}$ and $\mathrm{Ra}=10^{6}$. 


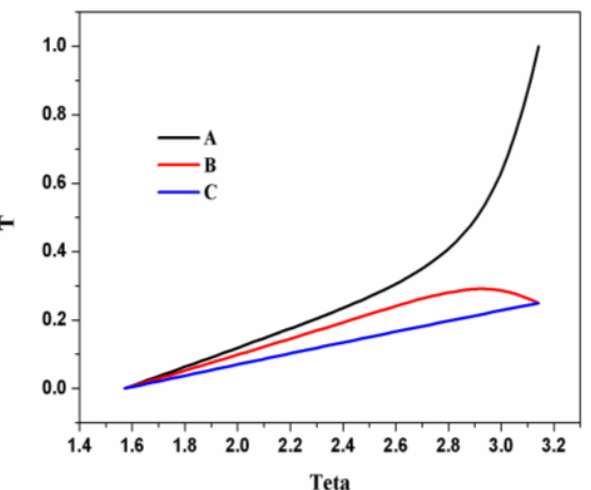

(a)

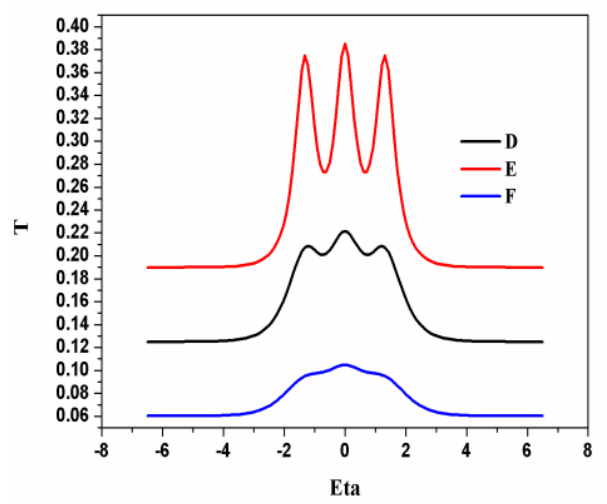

(b)
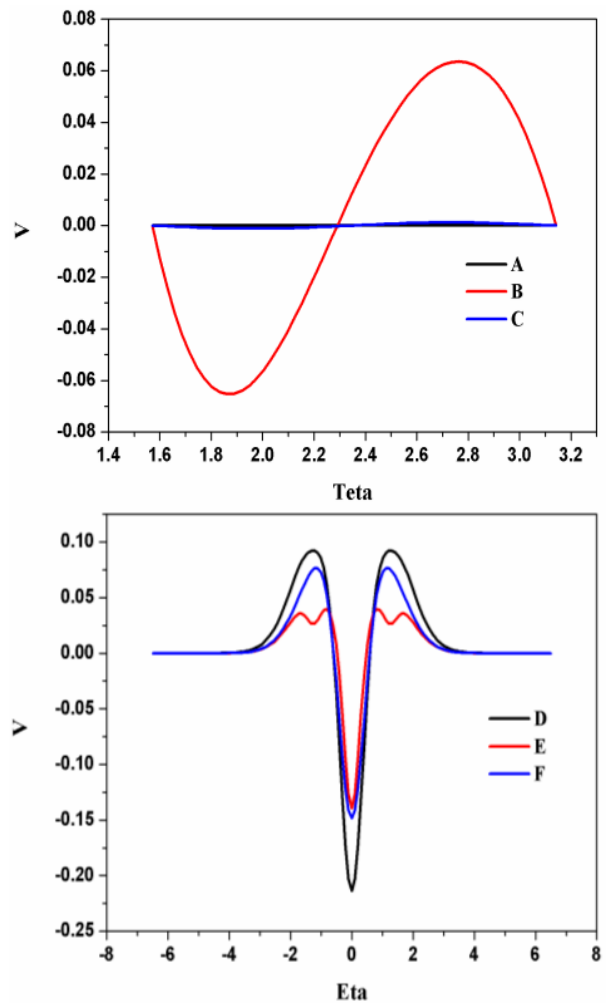

Figure 4. Vertical distribution of temperature and velocity along of the three axes for $\mathrm{Ra}=10^{3}$
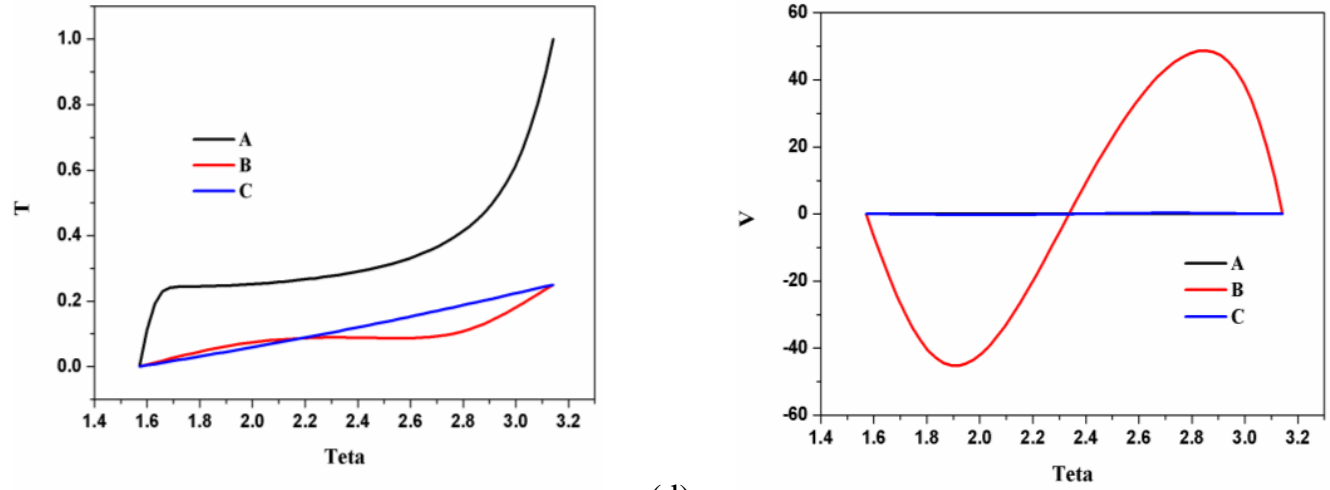

(c)

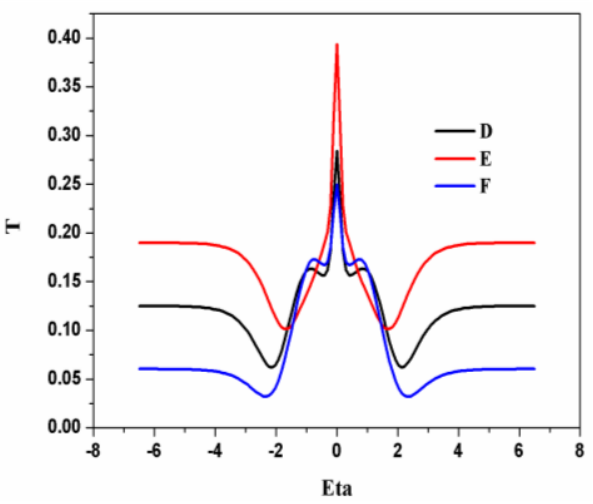

(d)

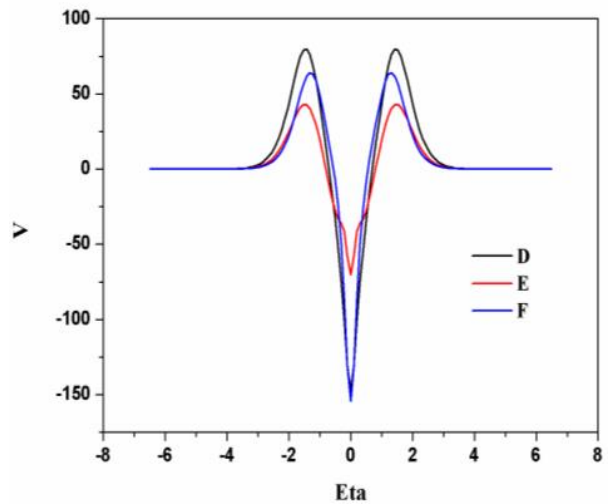

Figure 5. Horizontal distribution of temperature and velocity along of the three axes for $\mathrm{Ra}=10^{6}$

Figures (4.a) and (4.b) illustrate the vertical and horizontal temperature distribution (left) and velocities (right) respectively at $\mathrm{Ra}=10^{3}$. For the vertical temperature distribution, on axis $\mathrm{C}$, the profile is linear; and on axis $\mathrm{D}$, the profile is almost linear, showing that the conduction is favored (Figure (4a)). This is due to the closeness to the 
walls, but for axis A, the profile changes, leading to a decrease in the ready temperature of the hot wall, because of a low Rayleigh value. In addition, the profile of the horizontal temperatures is almost parallel and only deformed in the middle in the interval of Eta [-2.2]. This leads us to conclude that there is a stratification of air within the greenhouse, which means the presence of a pseudo-conductive regime. The horizontal velocities shown in Figure 4.b on all the axes have a remarkable sinusoidal motion in the interval of Eta [-3.3], reaching a maximum of 0.214 in dimensionless value. Nevertheless, outside this range, the velocity is almost zero because of the walls narrowing. In the vertical direction, the velocity is practically zero on all axes, except for axis $\mathrm{B}$, where a symmetrical movement with a maximum value of 0.063 can be seen.

On the other hand, Figures 5.a and Figure 5.b represent the vertical and horizontal temperatures distribution (left) and velocities (right), respectively for $\mathrm{Ra}=10^{6}$. The vertical temperature distribution: on $\mathrm{C}$ and $\mathrm{D}$ axes, the profile is almost linear like before, but more intense, showing that the conduction is favored. But for axis A, the profile changes, leading to a uniformly rapid decrease of temperature. These two configurations prove that convective heat transfer is intensifying, which implies an increase in vertical velocity on axis B up to a maximum value of (48.73). Additionally, as shown in Figure 5.b, the horizontal temperature is arching, moving away in height and the velocity is increasing to around 147.3, which means that the convection becomes more intense.

\section{The influence of tubes number on natural convection in the greenhouse}

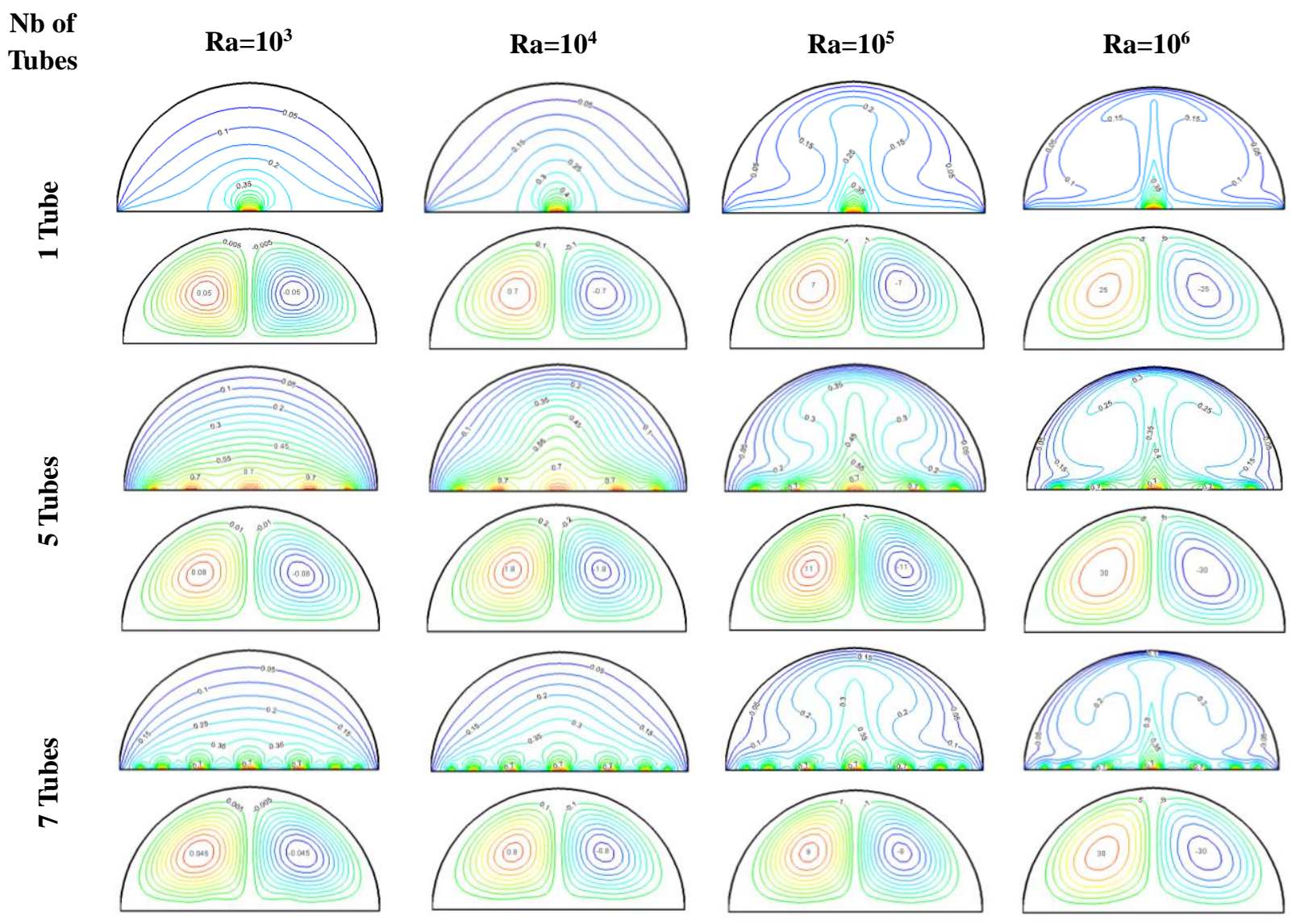

Figure 6. Isothermal and streamlines for different tubes and Rayleigh number

In this section, our main goal is to analyze the influence of the variation of the number of tubes $N t$ and the Rayleigh number $\mathrm{Ra}$ about the natural convection in the greenhouse (see Figure 6). Thence, curves for isotherms, streamlines, mean and local Nusselt number as well as vertical, horizontal temperature and velocity distributions are illustrated. 
The isotherms and streamlines are shown for various numbers of tubes $(1 \leq N t \leq 7)$ located in the bottom of greenhouse and for a Rayleigh number in the range $10^{3} \leq R a \leq 10^{6}$. We have seen that the shape of the arc largely explains the undulating profile of the air circulation inside the greenhouse, which directly affects the variations in air temperature and streamlines. In this section, we tried to study the consequences of changing the $N t$ number (1 tube up to 7 tubes) and for different Rayleigh numbers For a fixed number of tubes, it is noted that the air flow increases with increasing Rayleigh number. As well as for a large value of Ra equal to $10^{5}$ and the number $N t$ increases compared to the reference case, there is a noticeable change in both isothermal and streamlines curves, For low Rayleigh numbers, the isotherms are parallel, and become increasingly corrugated as the Rayleigh number increases. The increase in the number of Rayleigh induces an increase in buoyancy forces which means an increase in air velocity and therefore the extraction of a greater amount of heat, also the increase of the number of tubes plays a very important role on the natural convection in the greenhouse.

In addition, when $\mathrm{Nt}=1$ and $\mathrm{Ra}=10^{6}$, the isothermal lines change and end up adopting the shape of a mushroom, the temperature almost homogeneous in most of the interior volume of the greenhouse; whereas when more tubes are added, we notice that the convective transfer becomes more intense throughout the greenhouse.
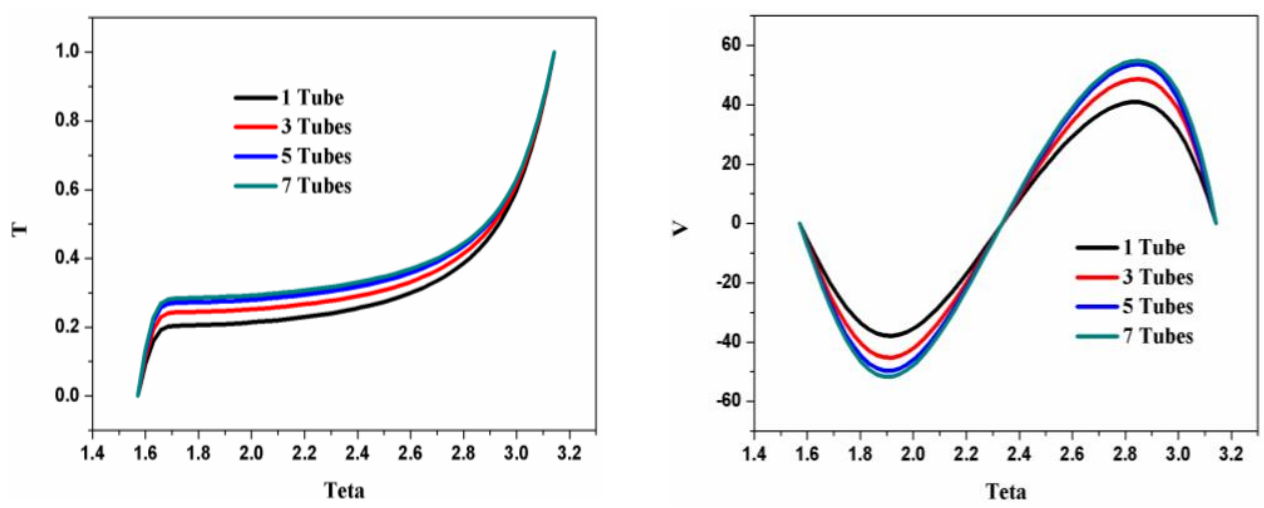

axis $\mathrm{A}$
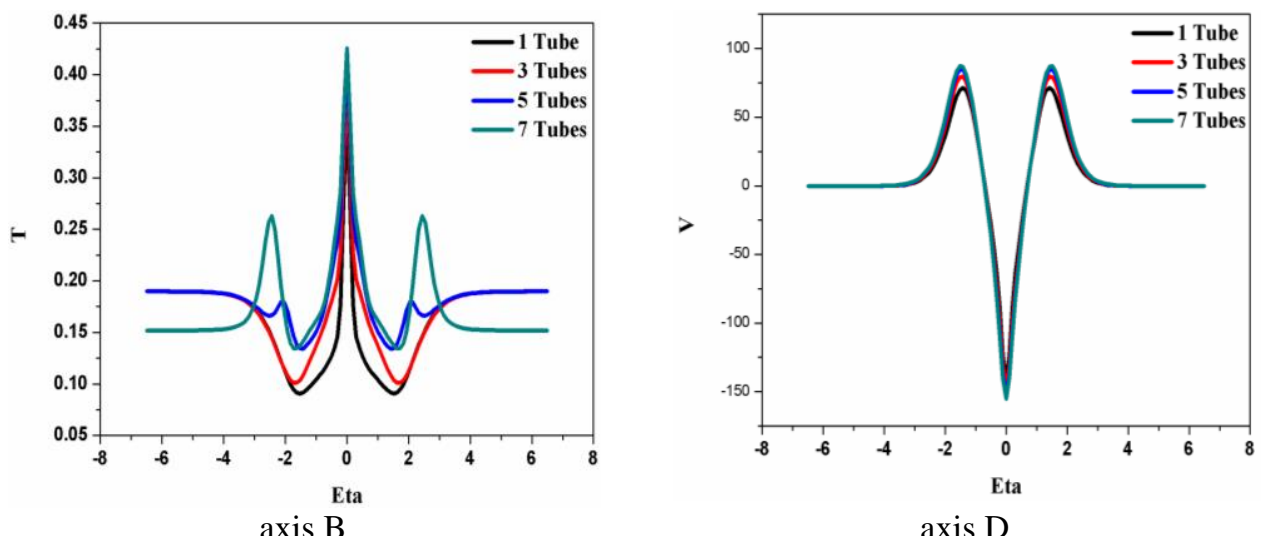

axis D

Figure 7. Distribution vertical and horizontal of the temperature and velocity for different tubes at $\mathrm{Ra}=10^{6}$

On axis A, the temperature profile decreases uniformly up to a height where the temperature continues to decrease in the neighboring area of the roof. This explains the tightening of the isothermal lines towards the top of the greenhouse as shown in Figure 4 and Figure 5. In the vertical direction, the velocity is maximum on the B axis; one can note a symmetrical movement with a maximum value for the case of 7 tubes when the velocity reaches a value of 54.91. Similarly, the profile of the horizontal temperatures is almost parallel and only deformed in the middle in the interval of Eta [-3.3], which translates the dominance of a convective regime flow. The horizontal velocities shown on axis D have a remarkable sinusoidal motion in the interval of Eta [-3.3] reaching a maximum of 155.3 in ultimate value. 


\section{Average and local Nusselt number}

The isotherms and streamlines structures (Figures. 6 and 7) do not provide exact information on the effect of the studied parameters ( $\mathrm{Ra}$ and $\mathrm{N} t$ ) on the total heat transfer inside the greenhouse. For this reason, the average Nusselt number and local Nusselt number are presented in figures 8 and 9 , respectively

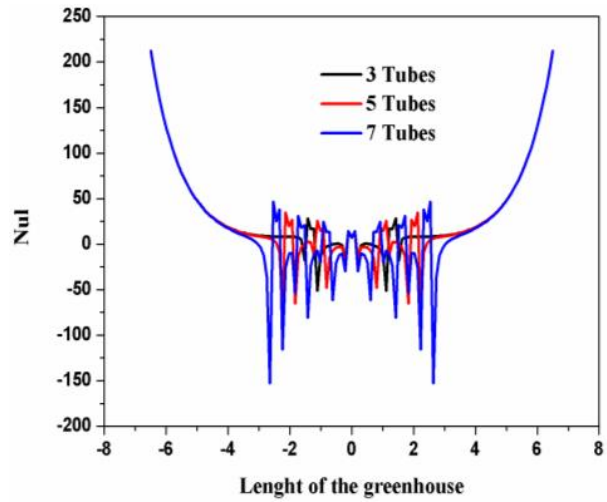

Figure 8. Variations of local Nusselt number in the hot wall

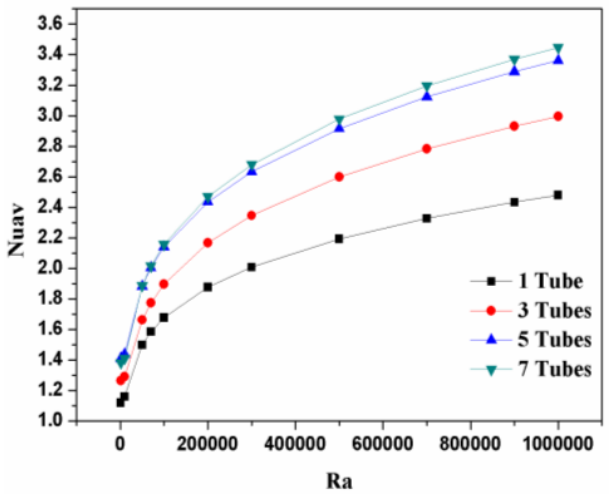

Figure 9. Variations of average Nusselt number according to the Ra and number of tubes

We have noticed, that there is a similarity between the local Nusselt profile for all the cases where the curve decreases at a certain vibratory range and then decreases for a maximum value (-152.57); the vibratory structure of this range caused by the air temperature near the bottom is higher than the average soil temperature. Also, the average Nusselt number is calculated by the summation of heat transfer flux in each node using the (eqs.19-20). For low Rayleigh number $\left(\mathrm{Ra}<10^{4}\right)$, the profile, is almost linear, and local Nusselt numbers are low, which shows that the heat transfer is dominated by pure conduction. With the increase of Rayleigh number $\left(\mathrm{Ra}>10^{4}\right)$, the natural convection becomes more important and heat exchange increases. In our simulation the correlation on average Nusselt number is given in Table 3:

Table 3. Correlation of average Nusselt number for differents configuration.

\begin{tabular}{|c|c|}
\hline 1 Tube & $0.8831+0.0201 R a^{0.3172}$ \\
\hline 3 Tubes & $1.0351+0.0119 R a^{0.3701}$ \\
\hline 5 Tubes & $1.1365+0.0158 R a^{0.3591}$ \\
\hline 7 Tubes & $1.0877+0.0174 R a^{0.3563}$ \\
\hline
\end{tabular}

\section{CONCLUSION}

We opted for a model based on the numerical resolution of natural convection inside a closed tunnel greenhouse, in laminar and permanent regime, using the vorticity-stream function formulation in bicylindrical coordinates, this model in 2D and is based on the assumption of Boussinesq. We have developed a numerical calculation code, based on a finite volume method, which allows to determine the dynamic and thermal fields in the greenhouse. The keys parameters of this simulation study the influence of Ra and Nt numbers on the dynamic field, thermal field and heat transfer in greenhouses. The results obtained in the form of streamlines, isotherms and Nusselt numbers. The most important conclusions drawn from this parametric study are as follows: 
- The results of numerical simulations have shown that conduction is the dominant heat transfer regime for Rayleigh numbers less than $10^{4}$ whereas natural convection prevails for Rayleigh numbers greater than $10^{4}$ for all treated cases.

- The effect of the number of tubes $\mathrm{N} t$ is also studied. It is finding that air flow and temperature inside the greenhouse are greatly influenced by the presence of the heated tubes. So, the heat transfer increases as the number of heating tubes increases.

- The average Nusselt number profile; for low Rayleigh numbers, it almost linear, showing that heat transfer exchanges are dominated by pure conduction. With the increase of Rayleigh number and the number of tubes number $\mathrm{N} t$, the natural convection becomes more dominant and the heat transfer increases.

- The percentage in average Nusselt number increases due to increasing number of heating tubes compared to Rayleigh number (see Table 4) :

Table 4. Comparison of the maximum heating tubes with the other cases in the average Nusselt number.

\begin{tabular}{|c|c|c|c|}
\hline \multirow{2}{*}{ Ra } & \multicolumn{3}{|c|}{$\begin{array}{c}\text { Percentage increase of the average Nusselt } \\
\text { number }(\%)\end{array}$} \\
\cline { 2 - 4 } & $(7-1)$ Tubes & $(7-3)$ Tubes & $(7-5)$ Tubes \\
\hline $10^{3}$ & 18.90 & 8.42 & 2.07 \\
\hline $10^{4}$ & 17.72 & 8.59 & 1.87 \\
\hline $10^{5}$ & 22.24 & 12.11 & 1.76 \\
\hline $5.10^{5}$ & 26.40 & 12.73 & 2.10 \\
\hline $10^{6}$ & 28.02 & 13.08 & 2.28 \\
\hline
\end{tabular}

With the increase in the number of tubes, the natural convection is more intense up to $\mathrm{N} t=7$. For $\mathrm{Ra}=10^{6}$, an optimum value of percentage improvement in heat transfer occurs when using tubeswhereas with 7 tubes, the exchange does not exceed $2 \%$. In this respect, using 5 tubes is shown to be more cost effective.

As a perspective, we intend to take into account: (i) numerical simulation of natural convection in a heated tunnel greenhouse (imposed flow), (ii) The effect of the height of the greenhouse on natural convection, (iii) numerical simulation of natural convection in an open tunnel greenhouse, (iv) numerical simulation of mixed convection in a closed and open tunnel greenhouse.

\section{NOMENCLATURE}

$\begin{array}{ll}a & \text { Focal distance, coefficient } \\ b & \text { Source term } \\ D & \text { Characteristic longer, } \mathrm{m} \\ F & \text { Function } \\ f & \text { Factor form } \\ G & \text { Function } \\ g & \text { Gravity acceleration, } \mathrm{m} . \mathrm{s}^{-2} \\ H & \text { Dimensionless metric Coefficient } \\ h & \text { Dimensional metric Coefficient, } \mathrm{m} \\ K & \text { Height of the greenhouse, } \mathrm{m} \\ \mathrm{L} & \text { Lenght of the greenhouse, } \mathrm{m} \\ \mathrm{NI} & \text { Maximal node number } \\ \mathrm{Nu} & \text { Nusselt number } \\ \mathrm{Nt} & \text { Tube number } \\ \mathrm{Pr} & \text { Prandtl number } \\ \mathrm{Ra} & \text { Rayleigh number } \\ T & \text { Dimensional temperature, } \mathrm{K}\end{array}$




\begin{tabular}{|c|c|}
\hline$u, v$ & Dimensional velocity-components, m. $\mathrm{s}^{-1}$ \\
\hline$x, y$ & Cartesian co-ordinates, $\mathrm{m}$ \\
\hline \multicolumn{2}{|l|}{ Greek symbols } \\
\hline$\alpha$ & Thermal diffusivity, $\mathrm{m}^{2} \mathrm{~s}^{-1}$ \\
\hline$\beta$ & Thermal expansion coefficient, $\mathrm{K}^{-1}$ \\
\hline$\varepsilon$ & Relative error \\
\hline$\eta$ & Vertical bi-cylindrical coordinate \\
\hline$\theta$ & Horizontal bi-cylindrical coordinate \\
\hline$v$ & Kinematic viscosity, $\mathrm{m}^{2} \mathrm{~s}^{-1}$ \\
\hline$\rho$ & Fluid density $\left[\mathrm{kgm}^{-3}\right], \mathrm{Kgm}^{-3}$ \\
\hline$\psi$ & Stream function, $\mathrm{s}^{-1}$ \\
\hline$\phi$ & Arbitrary variable \\
\hline$\omega$ & Vorticity, $\mathrm{s}^{-1}$ \\
\hline \multicolumn{2}{|l|}{ Subscripts } \\
\hline$a v$ & Average \\
\hline C & Cold \\
\hline$E$ & East \\
\hline$H$ & Hot \\
\hline$h$ & Dimensional metric symbol \\
\hline$l$ & Local \\
\hline $\mathrm{N}$ & North \\
\hline $\mathrm{P}$ & Principal \\
\hline S & South \\
\hline W & West \\
\hline$\eta$ & In vertical direction \\
\hline$\theta$ & In horizontal direction \\
\hline 0 & Reference state \\
\hline \multicolumn{2}{|l|}{ Superscripts } \\
\hline$n$ & Iteration number \\
\hline$*$ & Non-dimensional \\
\hline
\end{tabular}

\section{REFERENCES}

[1] U-Cheul Shin, Joseph Khedari, Cheikh Mbow, Michel Daguenet, Theoretical study of the natural convection in air-filled inclined enclosure, Int. J. Heat Mass Transfer,37 (1994), pp. 2007-2016.

[2] B. Draoui, M. Benyamine, Y.T.B. Tahri, Simulation Numérique de la Convection Naturelle en Régime Laminaire Transitoire dans une Serre Tunnel Chauffée par le Bas ( Flux ), (1999) 141-145.

[3] B.Draoui, M.Benyamine, R.Taïbi, O.Hami, Numerical Simulation in laminar unsteady natural Convection in Monochapelle Greenhouse heated from the bottom, Rev. Energ. Chemss (2000), pp. 67-73.

[4] N.S. Bondareva, M.A. Sheremet, Numerical simulation of natural convection melting in 2D and 3D enclosures, J. Therm. Eng. 5 (2019) 51-61. https://doi.org/10.18186/thermal.513015.

[5] Z. Altaç, N. Ugurlubilek, Two- and three-dimensional transient analysis of flow and heat transfer in structures with domical and curved roofs, J. Therm. Eng. 3 (2017) 1489-1497. https://doi.org/10.18186/journal-of-thermal-engineering.338895.

[6] A. Mezrhab, L. Elfarh, H. Naji, D. Lemonnier, Computation of surface radiation and natural convection in a heated horticultural greenhouse, Appl. $\quad$ Energy. $87 \quad$ (2010) 894-900. https://doi.org/10.1016/j.apenergy.2009.05.017.

[7] K.N. Cerci, E.K. Akpinar, Experimental determination of convective heat transfer coefficient during open sun and greenhouse drying of apple slices, J. Therm. Eng. 2 (2016) 741-747.

[8] H.F. Oztop, F. Selimefendigil, E. Abu-Nada, K. Al-Salem, Recent developments of computational methods on natural convection in curvilinear shaped enclosures. Journal of Thermal Engineering http://eds.yildiz.edu.tr/journal-of-thermal-engineering/Articles Yildiz Technical University Press, Istanbul, Turkey, (2016) 990-994. 
[9] Z.Kabdi, U-C.Shin, C.Mbowl, M.Daguenet, Laminar steady and two-dimensional thermal natural convection in cylindrical lunulas, Rev. Gén. Therm, 36 (1997), pp. 319-329.

[10] C.L. Chen, C.H. Cheng, Buoyancy-induced flow and convective heat transfer in an inclined arc-shape enclosure, Int. J. Heat Fluid Flow. 23 (2002) 823-830. https://doi.org/10.1016/S0142-727X(02)00189-3.

[11] T. Bartzanas, M. Tchamitchian, C. Kittas, Influence of the heating method on greenhouse microclimate and energy consumption, Biosyst. Eng. 91 (2005) 487-499. https://doi.org/10.1016/j.biosystemseng.2005.04.012.

[12] T. Nacima, Numerical simulation of natural convection in a horticultural greenhouse heated from below (by using CFD), Heat Transf. Res. 35 (2004) 302-335. https://doi.org/10.1615/HeatTransRes.v35.i34.130.

[13] N. Dihmani, H. Bouali, A.M.L. Elfarh, Simulation numérique des transferts thermiques dans une serre agricole chauffée par des blocs solides isothermes, Noûs. (2007) 221-224.

[14] S. Yildiz, Investigation of natural convection heat transfer at constant heat flux along a vertical and inclined plate, J. Therm. Eng. 4 (2018) 2432-2444. https://doi.org/10.18186/thermal.465654.

[15] W. Aich, L. Kolsi, M.N. Borjini, A.A.A.A. Al-Rashed, H. Ben Aissia, H.F. Oztop, N. Abu-Hamdeh, Threedimensional computational fluid dynamics analysis of buoyancy-driven natural ventilation and entropy generation in a prismatic greenhouse, Therm. Sci. 22 (2018) 73-85. https://doi.org/10.2298/TSCI151015052A.

[16] O. Yejjer, L. Kolsi, W. Aich, A.A.A.A. Al-Rashed, M.N. Borjini, H. Ben Aissia, Study of three-dimensional natural convection and entropy generation in an inclined solar collector equipped with partitions, Heat Transf. - Asian Res. 46 (2017) 1312-1326. https://doi.org/10.1002/htj.21275.

[17] W. Aich, I. Hajri, A. Omri, Numerical analysis of natural convection in a prismatic enclosure, Therm. Sci. 15 (2011) 437-446. https://doi.org/10.2298/TSCI1102437A.

[18] Patankar, S. V., Numerical Heat Transfer and Fluid Flow, Hemisphere., New York, USA, 1980.

[19] Nogotov, E.F., Applications of Numerical Heat Transfer, McGraw-Hill., New York, USA, 1978.

[20] A. Sharma, Introduction to Computational Fluid Dynamics, 2016. https://doi.org/10.1002/9781119369189. 